\title{
Serum soluble transferrin receptor levels are independently associated with homeostasis model assessment of insulin resistance in adolescent girls
}

\author{
Aleksandra Klisic ${ }^{1}$, Nebojsa Kavaric ${ }^{1}$, Jelena Kotur ${ }^{2}$, Ana Ninic ${ }^{2}$
}

\author{
${ }^{1}$ Primary Health Care Center, University of Montenegro, Faculty of Medicine, \\ Podgorica, Montenegro \\ ${ }^{2}$ Department for Medical Biochemistry, University of Belgrade, Faculty of Pharmacy, \\ Belgrade, Serbia
}

Submitted: 15 December 2020; Accepted: 24 January 2021

Publication online: 10 May 2021

Arch Med Sci

DOI: https://doi.org/10.5114/aoms/132757

Copyright $\odot 2021$ Termedia \& Banach

\begin{abstract}
Introduction: Markers of iron homeostasis are related to insulin resistance (IR) in adults. However, studies in children and adolescents are scarce and show contradictory results. The aim was to evaluate the potential relationship between iron status markers and IR. Additionally, no previous study has explored the mutual effect of biomarkers of iron homeostasis and inflammation (i.e. high sensitivity C-reactive protein (hsCRP)), and adipokines (i.e. retinol-binding protein 4 (RBP4)) on IR in the cohort of adolescent girls.

Material and methods: A total of 60 girls age between 16 and 19 years were included in the study. Serum levels of ferritin, transferrin, soluble transferrin receptor (STfR), hSCRP, and RBP4 were measured by immunonephelometry. Homeostasis model assessment of insulin resistance (HOMA-IR) and iron homeostasis indexes were calculated. Univariate and multivariate binary logistic regression analysis were used to investigate the possible independent associations of the examined biomarkers. Principal component analysis was used to examine their mutual effect on HOMA-IR in the studied girls.

Results: Ferritin, STfR, hSCRP and RBP4 were significant predictors for higher HOMA-IR in univariate analysis $(p=0.020, p=0.009, p=0.007, p=$ 0.003 , respectively). Multivariate regression analysis after adjustment for waist circumference (WC) showed that serum STfR levels remained positively associated with higher HOMA-IR $(p=0.044)$. Factorial analysis revealed that the obesity-inflammation related factor (i.e., WC and hsCRP) and adipokine-acute phase protein related factor (i.e., RBP4 and ferritin) showed significant differences between HOMA-IR $<2.5$ and HOMA-IR $\geq 2.5$.

Conclusions: Serum STfR levels are independently associated with HOMA-IR, whereas higher serum ferritin levels together with higher RBP4 are related to higher HOMA-IR in adolescent girls.
\end{abstract}

Key words: iron deficiency, cardiometabolic risk, adipokines, inflammation.

\section{Introduction}

Adolescence, as a period of increased nutritional demand and changes in psychosocial behavior, is often associated with obesity and metabolic disturbances closely related to insulin resistance (IR) [1]. IR may precede other cardiometabolic disorders, such as non-alcoholic fatty liver disease, metabolic syndrome, type 2 diabetes, and cardiovascular disease, later

\author{
Corresponding author: \\ Aleksandra Klisic \\ Primary Health \\ Care Center \\ Faculty of Medicine \\ University of Montenegro \\ Podgorica, Montenegro \\ Phone: +382 20481999 \\ E-mail: aleksandranklisic@ \\ gmail.com
}


in life [2-5]. In the aim of better understanding of the regulatory mechanisms of insulin signaling pathways, many biomolecules and their mutual interaction have been investigated [6-8]. Recent advances in that field indicated the relationship between several iron homeostasis biomarkers and IR [9-14]. However, those results are inconclusive, leaving doubt as to whether they act via different and independent pathways.

Ferritin has been widely used in iron deficiency anemia, and its low levels indicate depleted iron stores. However, it was suggested that ferritin may not be a reliable determinant of iron deficiency in individuals with obesity, since the obese state represents a bias factor when interpreting ferritin results [15]. Namely, as an acute phase reactant, ferritin levels increase in obesity, concomitantly with the increase in inflammation level and questioning the true reflection of iron stores [15, 16]. Transferrin is a protein that binds iron in the circulation, and its levels increase with increased requirement of iron. Similarly to ferritin, it increases in parallel with an increase in inflammation [9, 16]. The cellular demands of iron are regulated by the soluble transferrin receptors (sTfR). Those transmembrane receptors are presented in a soluble form in all cells. Through an interaction between transferrin and sTfR, cellular iron uptake occurs (i.e., transferrin-iron complex endocytosis), then STfR is released into the circulation $[9,16]$. Intracellular demands of iron and erythropoietic activity are known to be the principal regulators of sTfR expression. Therefore, its higher synthesis as well as higher circulating levels of STfR are increased in the case of iron deficiency. Unlike ferritin and transferrin, sTfR levels are considered not to be influenced by inflammation $[11,16]$.

Although the majority of studies on the associations between iron homeostasis biomarkers and IR were conducted in the adult population [9-11] and reported discrepant results, such research in youngsters is scarce [12-14], especially in late adolescents, as a vulnerable population group.

Hence, having in mind a smaller number of studies conducted in the pediatric population compared to adults, as well as the inconclusive results considering this issue, we aimed to gain a deeper knowledge into the complex pathophysiological mechanisms related to IR. Since inflammation and adipokines may also have an impact on insulin signaling pathways, in addition to several parameters of iron homeostasis (i.e., ferritin, transferrin, STfR and their indexes (sTfR/ferritin index and sTfR/log ferritin index)), we also included well-established inflammation biomarker (i.e. high sensitivity C-reactive protein (hSCRP)) and adipokine (i.e. retinol-binding protein 4 (RBP4)) to explore their joint effect on IR in the cohort of adolescent girls. Importantly, to our knowledge, no previous study has explored the mutual effect of adipokines, inflammation indices, and iron homeostasis parameters on IR.

\section{Material and methods}

\section{Study population}

A total of 60 adolescent girls who voluntarily agreed to participate were included in this case control study. The inclusion and exclusion criteria were described elsewhere [17]. In brief, girls older than 16 but younger than 19 years (i.e. girls from the third and fourth grade of two secondary schools in Podgorica) were included. Those girls who reported acute/chronic inflammatory disease, diabetes, hypothyroidism, hyperthyroidism, conditions that could affect body iron stores, as well as any medication used, were excluded. Those who reported being smokers or having consumed alcohol were also excluded to eliminate other potential bias factors related to inflammation.

The research was approved by the Ethical Committee of the Primary Health Care Center-Podgorica, Montenegro, following the ethical principles of the Helsinki Declaration. All girls provided signed informed consent. Parental written consent was also obtained for girls younger than 18 years.

\section{Anthropometric measurements}

Body weight (kg), waist circumference (WC) $(\mathrm{cm})$ and body height $(\mathrm{cm})$ were taken in the morning. WC was measured at the midpoint between the lowest rib and the iliac crest using tape over the abdomen without clothes. The non-stretchable tape was parallel to the floor without compressing the skin. Measurements were performed at the end of normal expiration. Body weight was measured to the nearest $0.1 \mathrm{~kg}$ on a balance beam scale. Girls were wearing light clothing and were without shoes. Height was measured to the nearest $0.1 \mathrm{~cm}$ using a stadiometer. For such purposes girls were also barefoot. Body mass index (BMI) was calculated as follows: weight $(\mathrm{kg})$ divided by height in meters squared $\left(\mathrm{kg} / \mathrm{m}^{2}\right)$ [18].

Adolescent girls with $30>\mathrm{BMI} \geq 25 \mathrm{~kg} / \mathrm{m}^{2}$ were considered to be overweight, whereas girls with $\mathrm{BMI} \geq 30 \mathrm{~kg} / \mathrm{m}^{2}$ were considered as obese. However, for simplicity we included all those girls in one group called the overweight/obese group (i.e. with $\mathrm{BMI} \geq 25 \mathrm{~kg} / \mathrm{m}^{2}$ ), whereas girls with $\mathrm{BMI}<25 \mathrm{~kg} / \mathrm{m}^{2}$ were regarded as normal weight.

\section{Biochemical analyses}

Venipuncture sampling was performed in the morning after an overnight fast of at least $8 \mathrm{~h}$, as previously stated [17]. Serum levels of RBP4, hsCRP, 
ferritin, transferrin, and STfR were measured immunonephelometrically (Behring Nephelometer Analyzer, Marburg, Germany). Standardized procedures were used for determination of serum levels of glucose, total protein, iron, and creatinine, and these analyses were measured on an automatic analyzer (Roche Cobas 400, Mannheim, Germany). Serum insulin was determined by chemiluminescent immunometric assay (AXSYM, Abbott, Abbott Park, Illinois, USA). Homeostasis model assessment of insulin resistance (HOMA-IR) [18], sTfR/ferritin index [19], and sTfR/log ferritin index [16] were calculated.

\section{Statistical analysis}

After testing the data distribution with the Shapiro-Wilk test, normally distributed continuous variables were expressed as the mean \pm standard deviation and skewed distributed continuous variables as medians and interquartile ranges. Comparisons between groups were analyzed by Student's $t$-test and the Mann-Whitney $U$ test dependent on data distribution. Categorical variables were presented as absolute frequencies. Non-parametric correlations between HOMA-IR and general demographic and laboratory data were tested with Spearman's correlation analysis. Those variables were given as a correlation coefficient $(\rho)$. Associations between HOMA-IR and tested variables were further analyzed by binary logistic regression analysis. The dependent cat- egorical variable was HOMA-IR given as follows: $0-$ HOMA-IR $<2.5$ and 1 - HOMA-IR $\geq 2.5$. Variables that were significantly correlated with HOMA-IR in Spearman's correlation analysis were afterwards analyzed in a multivariate binary regression analysis to determine their possible independent associations with HOMA-IR. The data from regression analyses were given as odds ratio (OR) and 95\% confidence intervals (Cls). Principal component analysis (PCA) [20] with varimax-normalized rotation was applied to cut the number of variables (which significantly correlated with HOMA-IR) in several significant factors. The criterion for factor extraction was its value $>1$, and factor loadings $>0.5$, and both were used for inclusion of variables. Finally, the number of factors was fixed at 3. This analysis revealed the scores for significant factors, which were used in the subsequent Mann-Whitney $U$ test to estimate the difference between factor groups related to lower vs. higher HOMA-IR values (i.e., $<2.5$ vs. $\geq 2.5$ ). $P$-values $<0.05$ were considered statistically significant. Statistical analysis was carried out using IBM SPSS Statistics version 22 software (IBM Corp., United States).

\section{Results}

Adolescent girls with HOMA-IR $\geq 2.5$ had higher $\mathrm{BMI}$ and WC than girls with HOMA-IR $<2.5$ (Table I). Moreover, they had significantly higher hsCRP, RBP4, transferrin, sTfR, and ferritin levels.

Table I. General demographic and laboratory data in adolescent girls

\begin{tabular}{|lccc|}
\hline Parameter & HOMA-IR $<2.5$ & HOMA-IR $\geq 2.5$ & $P$-value \\
\hline Adolescent girls, no. & 40 & 20 & \\
\hline Age $[$ [years] & $18(17-19)$ & $18(16-19)$ & 0.535 \\
\hline Weight $[\mathrm{kg}]$ & $64.5(60.0-70.5)$ & $78.5(68.5-96.0)$ & 0.002 \\
\hline Height $[\mathrm{cm}]$ & $169(166-173)$ & $168(165-177)$ & 0.900 \\
\hline BMI $\left[\mathrm{kg} / \mathrm{m}^{2}\right]$ & $22.6(20.1-25.5)$ & $27.5(25.2-31.5)$ & 0.001 \\
\hline WC $[\mathrm{cm}]$ & $79.0(73.5-87.0)$ & $96.0(87.5-103.5)$ & $<0.001$ \\
\hline Glucose $[\mathrm{mmol} /]^{*}$ & $5.05 \pm 0.37$ & $5.29 \pm 0.63$ & 0.066 \\
\hline Total proteins [g/l] & $73(71-74)$ & $73(70-74)$ & 0.624 \\
\hline hsCRP $[\mathrm{mg} / \mathrm{l}]$ & $0.44(0.23-0.91)$ & $1.10(0.56-2.05)$ & 0.002 \\
\hline RBP4 $[\mathrm{mg} / \mathrm{l}]$ & $28(26-32)$ & $35(29-41)$ & 0.004 \\
\hline Fe $[\mu \mathrm{mol} / \mathrm{l}]$ & $19.5(13.2-25.1)$ & $12.6(6.9-17.4)$ & 0.051 \\
\hline Transferrin $[\mathrm{g} /]^{*}$ & $2.53 \pm 0.35$ & $2.80 \pm 0.39$ & 0.008 \\
\hline sTfR $[\mathrm{mg} / \mathrm{dl}]^{*}$ & $12.08 \pm 2.37$ & $14.60 \pm 3.91$ & 0.003 \\
\hline Ferritin $[\mu \mathrm{h} / \mathrm{l}]$ & $23.8(19.7-30.8)$ & $36.2(20.0-40.9)$ & 0.047 \\
\hline sTfR/ferritin index & $0.49(0.35-0.61)$ & $0.37(0.32-0.63)$ & 0.355 \\
\hline sTfR/log ferritin index & $8.47(7.35-10.32)$ & $9.10(7.75-11.46)$ & 0.259 \\
\hline Insulin $[\mathrm{IU} / \mathrm{ml}]$ & $4.05(3.40-6.80)$ & $16.15(13.90-24.00)$ & $<0.001$ \\
\hline HOMA-IR & $0.90(0.78-1.53)$ & $3.44(3.09-5.95)$ & $<0.001$ \\
\hline
\end{tabular}

Data are shown as median (interquartile range). $P$ for Mann-Whitney $U$ test. ${ }^{*}$ Data are presented as mean \pm standard deviation. $P$ for Student's t-test. BMI - body mass index, WC - waist circumference, hSCRP - high-sensitivity C-reactive protein, RBP4 - retinol-binding protein 4, sTfR - soluble transferrin receptor, HOMA-IR - homeostasis model assessment of insulin resistance. 
A

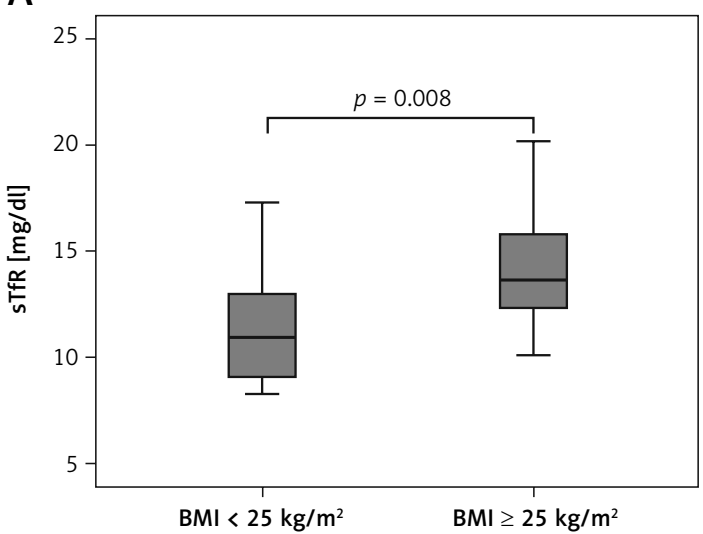

C

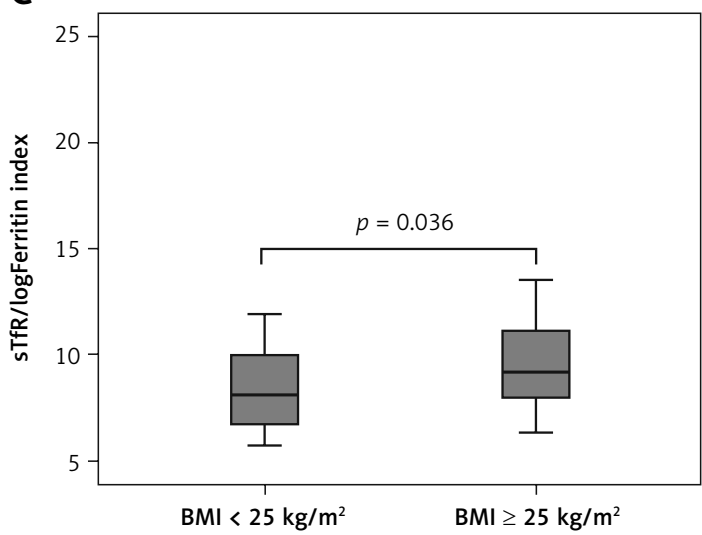

Furthermore, when girls were divided according to BMI, we observed significantly higher levels of sTfR and ferritin, as well as sTfR/log ferritin index in

Table II. Correlation coefficients of HOMA-IR with tested markers in examined female adolescents

\begin{tabular}{|lcc|}
\hline Parameter & $\rho$ & $P$-value \\
\hline Age [years] & -0.121 & 0.357 \\
\hline Weight $[\mathrm{kg}]$ & 0.594 & $<0.001$ \\
\hline Height $[\mathrm{m}]$ & -0.006 & 0.962 \\
\hline BMI $\left[\mathrm{kg} / \mathrm{m}^{2}\right]$ & 0.613 & $<0.001$ \\
\hline WC $[\mathrm{cm}]$ & 0.672 & $<0.001$ \\
\hline Glucose $[\mathrm{mmol} / \mathrm{l}]$ & 0.452 & $<0.001$ \\
\hline Total proteins [g/l] & -0.194 & 0.137 \\
\hline hsCRP $[\mathrm{mg} / \mathrm{l}]$ & 0.552 & $<0.001$ \\
\hline RBP4 $[\mathrm{mg} / \mathrm{l}]$ & 0.457 & $<0.001$ \\
\hline Fe $[\mu \mathrm{mol} / \mathrm{l}]$ & -0.215 & 0.138 \\
\hline Transferrin $[\mathrm{g} / \mathrm{l}]$ & 0.185 & 0.157 \\
\hline sTfR $[\mathrm{mg} / \mathrm{dl}]$ & 0.361 & 0.005 \\
\hline Ferritin $[\mu \mathrm{g} / \mathrm{l}]$ & 0.360 & 0.005 \\
\hline sTfR/ferritin index $[\mathrm{mg} / \mu \mathrm{\mu g}]$ & -0.181 & 0.167 \\
\hline sTfR/log ferritin index & 0.142 & 0.279 \\
\hline
\end{tabular}

Data age given as coefficients of correlation rho ( $\rho)$. BMI - body mass index, WC - waist circumference, hSCRP - high-sensitivity $C$-reactive protein, RBP4 - retinol-binding protein 4, sTfR - soluble transferrin receptor, HOMA - IR-homeostasis model assessment of insulin resistance.
B

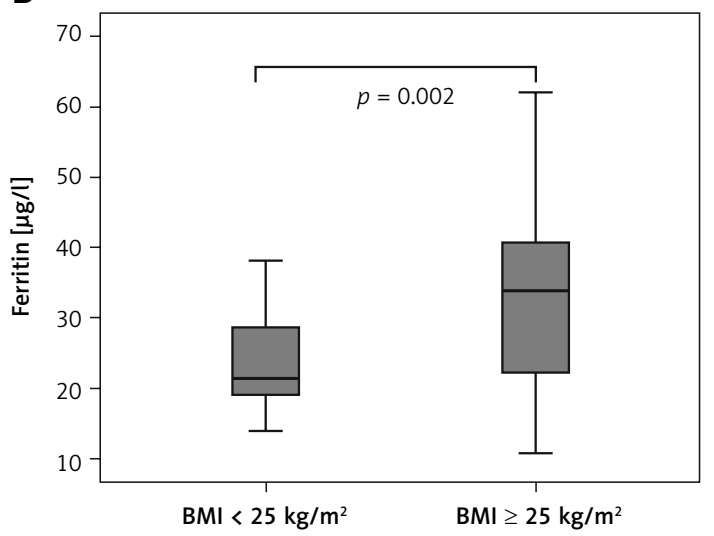

Figure 1. Iron status markers in normal weight $\left(\mathrm{BMI}<25 \mathrm{~kg} / \mathrm{m}^{2}\right)$ and overweight/obese adolescent girls $\left(\mathrm{BMI} \geq 25 \mathrm{~kg} / \mathrm{m}^{2}\right)$

the overweight/obese group (i.e., $\mathrm{BMI} \geq 25 \mathrm{~kg} / \mathrm{m}^{2}$ ) ( $p=0.008, p=0.002, p=0.036$, respectively), compared to normal weight counterparts (i.e., $\mathrm{BMI}<25 \mathrm{~kg} / \mathrm{m}^{2}$ ) (Figure 1).

In contrast, no significant difference in transferrin and STfR/Ferritin index between these groups was observed (data not presented).

Correlation coefficients given in Table II revealed significant positive correlations between HOMA-IR and weight, BMI, WC, glucose, hsCRP, RBP4, sTfR, and ferritin.

We further evaluated the ability of inflammation and iron status markers to discriminate between HOMA-IR groups (Table III). Predictors that were significantly correlated with HOMA-IR in Spearman's correlation analysis were tested by binary regression analysis. Ferritin, STfR, hsCRP, and RBP4 were significant predictors of higher HOMA-IR (Table III). Nagelkerke $R^{2}$ [21] revealed that each predictor in univariate analysis (hsCRP, RBP4, STfR, and ferritin) could explain the variation in HOMA-IR by $19.5 \%, 24.1 \%, 19.0 \%$ and $13.5 \%$, respectively. Additionally, independent associations of predictors of HOMA-IR by multivariate regression analysis were assessed. Firstly, we performed an adjustment for WC as a marker highly related to HOMA-IR (Model 1). Only STfR levels remained positively associated with higher HOMA-IR. Finally, all predictors and WC were in- 
Table III. Univariate and multivariate binary logistic regression analysis for the associations of laboratory data and HOMA-IR in female adolescents

\begin{tabular}{|c|c|c|c|}
\hline Predictors & $\begin{array}{l}\text { Unadjusted } \\
\text { OR }(95 \% \mathrm{Cl})\end{array}$ & $P$-value & $R^{2}$ \\
\hline hsCRP [mg/l] & $2.671(1.302-5.481)$ & 0.007 & 0.195 \\
\hline RBP4 [mg/l] & $1.167(1.054-1.292)$ & 0.003 & 0.241 \\
\hline $\mathrm{sTfR}[\mathrm{mg} / \mathrm{dl}]$ & $1.330(1.074-1.647)$ & 0.009 & 0.190 \\
\hline Ferritin $[\mu \mathrm{g} / \mathrm{I}]$ & $1.067(1.010-1.127)$ & 0.020 & 0.135 \\
\hline Model 1 & $\begin{array}{c}\text { Adjusted } \\
\text { OR }(95 \% \mathrm{Cl})\end{array}$ & $P$-value & $R^{2}$ \\
\hline $\mathrm{hsCRP}[\mathrm{mg} / \mathrm{l}]$ & $1.136(0.425-3.038)$ & 0.799 & 0.336 \\
\hline RBP4 [mg/l] & $1.118(1.000-1.250)$ & 0.050 & 0.410 \\
\hline $\mathrm{sTfR}[\mathrm{mg} / \mathrm{dl}]$ & $1.259(1.006-1.577)$ & 0.044 & 0.357 \\
\hline Ferritin $[\mu \mathrm{g} / \mathrm{l}]$ & $1.034(0.975-1.097)$ & 0.266 & 0.413 \\
\hline Model 2 & $\begin{array}{c}\text { Adjusted } \\
\text { OR }(95 \% \mathrm{Cl})\end{array}$ & $P$-value & $R^{2}$ \\
\hline $\mathrm{hsCRP}[\mathrm{mg} / \mathrm{l}]$ & $1.006(0.345-2.932)$ & 0.991 & 0.487 \\
\hline RBP4 [mg/l] & $1.094(0.972-1.231)$ & 0.138 & \\
\hline $\mathrm{sTfR}[\mathrm{mg} / \mathrm{dl}]$ & $1.288(1.012-1.641)$ & 0.040 & \\
\hline Ferritin $[\mu g / l]$ & $1.034(0.959-1.144)$ & 0.338 & \\
\hline
\end{tabular}

Model 1: each marker adjusted for WC. Model 2: consisted of all tested markers and WC. hSCRP-high-sensitivity C-reactive protein, RBP4 - retinol-binding protein 4, sTfR - soluble transferrin receptor, HOMA-IR - homeostasis model assessment of insulin resistance.

cluded in Model 2. STfR retained its independence in the prediction of higher HOMA-IR. Explained variation of Model 2 in HOMA-IR was close to $50 \%$.

PCA was performed to obtain groups of a smaller number of factors related to the same level of variability. It extracted the larger number of biomarkers which significantly correlated with HOMA-IR.

This analysis emphasized 3 different factors explaining $66 \%$ of variance of the tested parameters (Table IV). The largest percent of variance (38\%) was shown by the obesity-inflammation related factor with positive loadings of WC and hsCRP. The second factor explained $17 \%$ of the variation and consisted of iron homeostasis parameters (i.e., transferrin and STfR, both with positive loadings), and the adipokine-acute phase protein related factor explained $11 \%$ of the variation (i.e., RBP4 and ferritin, both parameters with positive loadings).

Mann-Whitney $U$ test enabled us to estimate the difference between factor groups related to lower vs. higher HOMA-IR values. Our analysis showed that the obesity-inflammation related factor and adipokine-acute phase protein related factor significantly differentiated between lower and higher HOMA-IR values (Table V).

\section{Discussion}

As far as we know, the current study is the first to investigate the mutual influence of adipokine (i.e. RBP4), inflammation biomarker (i.e. hsCRP), and iron homeostasis indices on HOMA-IR to get a better insight into the IR status through multiple different pathways. Moreover, it is one of the few to examine iron homeostasis parameters in relation to IR (as determined with HOMA-IR) in an exclusively late adolescent population.

Univariate analysis in our current study revealed that ferritin, STfR, hsCRP, and RBP4 were significant predictors of HOMA-IR (Table III). However, after multivariate regression analysis, only sTfR levels were independently correlated with HOMA-IR. This relationship was independent of

Table IV. Principal component analysis of extracted factors connected with HOMA-IR

\begin{tabular}{|lcc|}
\hline Factors & Included variables with loadings & Factor variability \\
\hline Obesity-inflammation related factor & WC $(0.912)$ & $38 \%$ \\
\hline Iron homeostasis related factor & hSCRP $(0.780)$ & $17 \%$ \\
\hline Adipokine-acute phase protein related factor & $\begin{array}{c}\text { Transferrin }(0.875) \\
\text { STfR }(0.728)\end{array}$ & $11 \%$ \\
\hline
\end{tabular}

$W C$ - waist circumference, hSCRP - high-sensitivity C-reactive protein, sTfR - soluble transferrin receptor, RBP4-retinol-binding protein 4, HOMA-IR - homeostasis model assessment of insulin resistance. 
Table V. Mann-Whitney $U$ test for the difference between factor groups related to higher HOMA-IR values

\begin{tabular}{|lc|}
\hline Factors & $\begin{array}{c}P \text { for the difference between HOMA-IR }<2.5 \\
\text { and HOMA-IR } \geq 2.5\end{array}$ \\
\hline Obesity-inflammation related factor (WC and hsCRP) & 0.004 \\
\hline Iron homeostasis related factor (transferrin and STfR) & 0.347 \\
\hline Adipokine-acute phase protein related factor (RBP4 and ferritin) & 0.006 \\
\hline WC - waist circumference, hsCRP - high-sensitivity C-reactive protein, sTfR - soluble transferrin receptor, RBP4 - retinol-binding protein 4, \\
HOMA-IR - homeostasis model assessment of insulin resistance.
\end{tabular}

obesity status, since adjustment for WC was performed (Table III, Model 1).

Our results of the relationship between sTfR and HOMA-IR are in accordance with several studies in European adult populations [9, 10]. The meta-analysis of Liu et al. [11] that included 12 cohort and case-control studies showed that higher serum ferritin level was related to the risk of type 2 diabetes, whereas STfR/ferritin ratio was inversely associated with the risk of type 2 diabetes. Similar findings (i.e., an inverse relationship between sTfR/ferritin level and HOMA-IR) were obtained in Austrian adolescent girls, but not in boys of similar age [12].

Despite the relatively small sample size in our study, we included only normal weight and overweight/obese girls of a narrow age range (between 16 and 19 years) who reported no other diseases, as well as no medications, tobacco or alcohol use. Therefore, we aimed to diminish any potential confounding factors that might have an influence on inflammation level and IR. In contrast, a study conducted in a young population with obesity and with a smaller sample size than ours reported no difference in STfR levels between patients with impaired glucose tolerance/type 2 diabetes and the corresponding control group [13]. The authors of the mentioned study presumed that the degree of obesity, rather than impaired glycemia, is the determinant of sTfR levels in circulation.

However, in a recent large cohort of Chinese children and adolescents between 7 and 18 years of age, except for ferritin, no difference in STfR and transferrin levels between overweight/obese and non-obese children was reported. Interestingly, these markers of iron homeostasis (i.e., transferrin and STfR) were negatively associated with the risk of dyslipidemia in the examined youngsters [14]. Another earlier study that encompassed Chinese children and adolescents of a similar age range as the latter one reported that higher serum transferrin was positively associated with HOMA-IR, but no such relationships between HOMA-IR and ferritin or sTfR levels were found. Surprisingly, they found an inverse association between STfR and glycated hemoglobin $\left(\mathrm{HbA}_{1 \mathrm{c}}\right)$ [22].

Similarly, a study that included Korean children also reported no association of serum STfR and ferritin levels with HOMA-IR [23].
Although ours is the first study to report an independent association between STfR and HOMA-IR in the adolescent population, our results are comparable with those in adults. Namely, in a cohort of adults with obesity, it was found that increased STfR levels are related to an increased risk for type 2 diabetes occurrence [24]. Indeed, a relationship between polymorphisms of transferrin receptor genes (rs3817672, 210AG, S142G) and type 2 diabetes was found [25]. Moreover, Suárez-Ortegón et al. [9] in a relatively large sample of Croatian population found no association between sTfR and metabolic syndrome components, except for HOMA-IR. Similarly, the results of the study of Huth et al. [10] in a large sample of German adults reported an association between STfR and HOMA-IR, suggesting that high insulin levels might cause higher STfR. In line with this, a significant decrease in STfR levels in parallel with reversing insulin resistance after an exercise program in obese women was reported [26]. In addition, it was found that upregulation of erythropoiesis and the consequent increase in STfR were influenced by insulin [27].

To further clarify the association between obesity-related inflammation and iron homeostasis indices with IR, we included in the analysis hsCRP and RBP4, biomarkers that are increased in the obese state $[28,29]$. Indeed, we reported their independent association with HOMA-IR in the current study. When we included all predictors (hsCRP, RBP4, ferritin, and STfR, as well as WC) in the multivariate regression analysis, STfR still retained its independence in the prediction of HOMA-IR (Table III, Model 2). The PCA in our study revealed 3 group factors related to HOMA-IR. The largest percent of variance (38\%) was shown by the obesity-inflammation related factor (i.e., WC and hs(RP), followed by the second factor, the iron homeostasis parameters related factor (i.e., transferrin and STfR), which explained $17 \%$ of the variation and the third, the adipokine-acute phase protein related factor (i.e., RBP4 and ferritin), which explained $11 \%$ of the variation (Table IV). However, only the first (i.e., WC and hsCRP) and the third group factor (i.e., RBP4 and ferritin) showed significant difference between HOMA-IR $<2.5$ and HOMA-IR $\geq 2.5$ (Table V). On the other hand, among the 3 examined iron status biomark- 
ers (i.e., ferritin, transferrin and STfR), transferrin was the weakest predictor of HOMA-IR. This may explain why the iron homeostasis parameter related factor (which included the joint effect of transferrin and STfR) did not differentiate between HOMA-IR $<2.5$ and HOMA-IR $\geq 2.5$.

HsCRP and ferritin levels are regarded as acutephase reactants, which are found to be higher in the obesity-related inflammatory state [15, $28,30]$. In line with this, a positive association between STfR and CRP was reported previously [31]. Another possible explanation of our findings might involve serum levels of hepcidin (i.e., a liverand adipose tissue-derived acute phase reactant), which are increased in obesity [15]. Since hepcidin regulates iron homeostasis, it is speculated that its increased expression in obesity inhibits iron absorption in the intestine and mobilization from stores in the liver, with consequent reduced iron levels in the circulation and increased iron stores [15]. The proposed mechanism is that obesity-induced inflammation enhances the expression of hepcidin through the interleukin-6-signal transducer and activator of transcription-3 pathway [32], thus reducing iron levels and consequently increasing ferritin and STfR in circulation [15].

Discordant results in previous studies, in addition to the variation in sample size, gender distribution, and ethnic background, might also be attributed to different assays for measurement of sTfR and other iron homeostasis markers (e.g., immunoturbidimetry, immunonephelometry, electrochemiluminescence assays) [9, 10, 32]. Since no assay standardization for STfR exists and regarding the presence of its different cut-off levels, the interpretation of STfR in clinical practice is limited $[33,34]$. Therefore, a better understanding of the factors that modulate STfR is of great importance in future studies.

Besides the small sample size and cross-sectional nature of this study, there is another limitation that needs to be mentioned. Namely, like many previous studies, we were limited in the dietary intake assessment, since it could affect, at least in part, iron stores and examined iron homeostasis markers. However, we excluded many bias factors that could influence inflammation status, as previously mentioned. Further studies with a larger sample size and both genders included, as well as with a longitudinal design, are needed to validate our results and to examine the causal relationship between the indices of iron homeostasis and insulin resistance. Better insights into the pathophysiological processes of iron deficiency status and obesity-related disturbances are needed to find the most reliable therapeutic approach to the consequences of obesity.

In conclusion, this is the first study to examine the mutual influence of adipokine (i.e., RBP4), an inflammation biomarker (i.e., hsCRP), and iron homeostasis indices on HOMA-IR. It is also the first one in the adolescent population to report a relationship between STfR and HOMA-IR, as a surrogate marker of insulin resistance. This relationship is independent of obesity (as determined by WC). Moreover, factorial analysis showed that the obesity-inflammation related factor (i.e., WC and hs(RP) and adipokine-acute phase protein related factor (i.e., RBP4 and ferritin) showed significant difference between HOMA-IR $<2.5$ and HOMA-IR $\geq 2.5$.

\section{Acknowledgments}

This work was financially supported in part by a grant from the Ministry of Science, Montenegro and the Ministry of Education, Science and Technological Development, Republic of Serbia (project number 451-03-68/2020-14/200161).

\section{Conflict of interest}

The authors declare no conflict of interest.

\section{References}

1. Das JK, Salam RA, Thornburg KL, et al. Nutrition in adolescents: physiology, metabolism, and nutritional needs. Ann N Y Acad Sci 2017; 1393: 21-33.

2. Čolak E, Pap D. The role of oxidative stress in the development of obesity and obesity-related metabolic disorders. J Med Biochem 2021; 40: 1-9.

3. Klisic A, Kavaric N, Ninic A. Serum uric acid, triglycerides and total bilirubin are associated with Hepatic Steatosis Index in adolescent population. Preventivna Pedijatrija 2020; 6: 71-6.

4. Čolak E, Pap D, Nikolić LJ, Vicković S. The impact of obesity to antioxidant defense parameters in adolescents with increased cardiovascular risk. J Med Biochem 2020; 39: 346-54.

5. Maksimovic M, Vlajinac H, Radak DJ, Marinkovic J, Maksimovic J, Jorga J. Association of overweight and obesity with cardiovascular risk factors in patients with atherosclerotic diseases. J Med Biochem 2020; 38: 215-23.

6. Klisic A, Kavaric N, Stanisic V, et al. Endocan and a novel score for dyslipidemia, oxidative stress and inflammation (DOI score) are independently correlated with glycated hemoglobin ( $\mathrm{HbA} 1 \mathrm{c})$ in patients with prediabetes and type 2 diabetes. Arch Med Sci 2020; 16: 42-50.

7. Sayın S, Kutlu R, Kulaksızoğlu M. The relationship between sex steroids, insulin resistance and body compositions in obese women: a case-control study. J Med Biochem 2020; 39: 25-31.

8. Klisic A, Kavaric N, Vujcic S, et al. Inverse association between serum endocan levels and small LDL and HDL particles in patients with type 2 diabetes mellitus. Eur Rev Med Pharmacol Sci 2020; 24: 8127-35.

9. Suárez-Ortegón MF, McLachlan S, Wild SH, Fernández-Real JM, Hayward C, Polašek O. Soluble transferrin receptor levels are positively associated with insulin resistance but not with the metabolic syndrome or its individual components. Br J Nutr 2016; 116: 1165-74.

10. Huth C, Beuerle S, Zierer A, et al. Biomarkers of iron metabolism are independently associated with impaired 
glucose metabolism and type 2 diabetes: the KORA F4 study. Eur J Endocrinol 2015; 173: 643-53.

11. Liu J, Li Q, Yang Y, Ma L. Iron metabolism and type 2 diabetes mellitus: a meta-analysis and systematic review. J Diabetes Investig 2020; 11: 946-55.

12. Aigner E, Hinz C, Steiner K, et al. Iron stores, liver transaminase levels and metabolic risk in healthy teenagers. Eur J Clin Invest 2010; 40: 155-63.

13. Shalitin S, Deutsch V, Tauman R. Hepcidin, soluble transferrin receptor and IL-6 levels in obese children and adolescents with and without type 2 diabetes mellitus/ impaired glucose tolerance and their association with obstructive sleep apnea. J Endocrinol Invest 2018; 41: 969-75.

14. Zhu Y, He B, Xiao Y, Chen Y. Iron metabolism and its association with dyslipidemia risk in children and adolescents: a cross-sectional study. Lipids Health Dis 2019; 18: 50 .

15. Hutchinson C. A review of iron studies in overweight and obese children and adolescents: a double burden in the young? Eur J Nutr 2016; 55: 2179-97.

16. Lopez A, Cacoub P, Macdougall IC, Peyrin-Biroulet L. Iron deficiency anaemia. Lancet 2016; 387: 907-16.

17. Klisic A, Kavaric N, Ninic A. Serum cystatin C levels are associated with triglycerides/high-density lipoprotein cholesterol ratio in adolescent girls ages between 1619 years old. Eur Rev Med Pharmacol Sci 2020; 24: 10680-6.

18. Klisic A, Kavaric N, Jovanovic M, Soldatovic I, Gligorovic-Barhanovic N, Kotur-Stevuljevic J. Bioavailable testosterone is independently associated with fatty liver index in postmenopausal women. Arch Med Sci 2017; 5: 1188-96.

19. Skikne BS, Flowers CH, Cook JD. Serum transferrin receptor. A quantitative measure of tissue iron deficiency. Blood 1990; 75: 1870-6.

20. Abdi H, Williams LJ. Principal Component Analysis. Wiley Interdisciplinary Reviews: Computational Statistics 2010; 2: 433-59.

21. Nagelkerke NJD. A note on the general definition of the coefficient of determination. Biometrika 1991; 78: 691-2.

22. Wei J, Luo X, Zhou S, et al. Associations between iron status and insulin resistance in Chinese children and adolescents: findings from the China Health and Nutrition Survey. Asia Pac J Clin Nutr 2019; 28: 819-25.

23. Lee HJ, Jang HB, Park JE, et al. Relationship betwen serum levels of body iron parameters and insulin resistance and metabolic syndrome in Korean Children. Osong Public Health Res Perspect 2014; 5: 204-10.

24. Fernández-Cao JC, Arija V, Aranda N, et al. Soluble transferrin receptor and risk of type 2 diabetes in the obese and nonobese. Eur J Clin Invest 2017; 47: 221-30.

25. Fernández-Real JM, Mercader JM, Ortega FJ, et al. Transferrin receptor-1 gene polymorphisms are associated with type 2 diabetes. Eur J Clin Invest 2010; 40: 600-7.

26. Fernández-Real JM, Izquierdo M, Moreno-Navarrete JM, et al. Circulating soluble transferrin receptor concentration decreases after exercise-induced improvement of insulin sensitivity in obese individuals. Int J Obes 2009; 33: 768-74.

27. Ratajczak J, Zhang Q, Pertusini E, et al. The role of insulin (INS) and insulin-like growth factor-I (IGF-I) in regulating human erythropoiesis. Studies in vitro under serum-free conditions: comparison to other cytokines and growth factors. Leukemia 1998; 12: 371-81.

28. Klisic A, Kavaric N, Soldatovic I, Bjelakovic B, KoturStevuljevic J. Relationship between cardiovascular risk score and traditional and nontraditional cardiometabol ic parameters in obese adolescent girls. J Med Biochem 2016; 35: 282-92.

29. Olsen T, Blomhoff R. Retinol, retinoic acid, and retinol-binding protein 4 are differentially associated with cardiovascular disease, type 2 diabetes, and obesity: an overview of human studies. Adv Nutr 2020; 11: 644-66.

30. Wang YS, Ye J, Yang X, et al. Association of retinol binding protein-4, cystatin $C$, homocysteine and high-sensitivity C-reactive protein levels in patients with newly diagnosed type 2 diabetes mellitus. Arch Med Sci 2019; 15: $1203-16$.

31. Turgeon O’Brien H, Blanchet R, Gagné D, Lauzière J, Vézina C. Using soluble transferrin receptor and taking inflammation into account when defining serum ferritin cutoffs improved the diagnosis of iron deficiency in a group of canadian preschool inuit children from Nunavik. Anemia 2016; 2016: 6430214.

32. Verga Falzacappa MV, Vujic Spasic M, Kessler R, Stolte J, Hentze MW, Muckenthaler MU. STAT3 mediates hepatic hepcidin expression and its inflammatory stimulation. Blood 2007; 109: 353-8.

33. Pfeiffer CM, Looker AC. Laboratory methodologies for indicators of iron status: strengths, limitations, and analytical challenges. Am J Clin Nutr 2017; 106: 1606S-14S.

34. Vázquez-López MA, Ibáñez-Alcalde $M$, Lendínez-Molinos F, et al. Reference values of serum transferrin receptor and STfR/log ferritin index in healthy adolescents. J Pediatr Hematol Oncol 2015; 37: 274-80. 